\section{Correlación entre nivel de conocimientos sobre posturas odontológicas ergonómicas, posturas de trabajo y dolor postural según zonas de respuesta, durante las prácticas clínicas de estudiantes en una Facultad de Estomatología}

Bendezú NV, Valencia E, Aguilar LA, Vélez C. Correlación entre nivel de conocimientos sobre posturas odontológicas ergonómicas, posturas de trabajo y dolor postural según zonas de respuesta, durante las prácticas clínicas de estudiantes en una Facultad de Estomatología. Rev Estomatol Herediana 2006; 16(1): 26 - 32.

\section{RESUMEN}

En el presente estudio se investigaron los aspectos ergonómicos y dolor postural aplicados a la actividad odontológica, además de proporcionar información en busca de una buena calidad de vida y capacidad productiva en el campo de la salud ocupacional del Odontólogo. El objetivo fue determinar la correlación entre nivel de conocimientos sobre posturas odontológicas ergonómicas, posturas de trabajo y presencia de dolor postural según zonas anatómicas de respuesta durante las prácticas clínicas del estudiante del quinto año de la Facultad de Estomatología de la Universidad Peruana Cayetano Heredia. Se utilizaron: cuestionario de conocimientos en relación a posturas de trabajo ergonómicas, lista de verificación postural que comprobó la postura de trabajo clínico del estudiante, apoyada por fotografías digitales analizadas por medio de los programas de computación Autocad y Corel Draw. La Escala Analógica Visual (EAV) de percepción e intensidad del dolor postural según zonas anatómicas de respuesta. Para el análisis estadístico se utilizó el coeficiente de correlación de rangos de Spearman. En cuanto a las observaciones posturales de trabajo odontológico, sólo el 22,3\% fueron correctas. Del universo de preguntas sobre posturas odontológicas, sólo 90 (37,5\%) fueron respondidas correctamente. La percepción de dolor postural fue mayor en la zona cervical (75\%) y menor en antebrazos (15\%). Se encontró correlación entre nivel de conocimientos sobre posturas odontológicas ergonómicas y la aplicación de posturas de trabajo odontológico. Se concluye que existe relación directa entre las variables estudiadas.

Palabras clave: INGENIERÍA HUMANA / SALUD LABORAL / ESTUDIANTES DE ODONTOLOGÍA / CONOCIMIENTOS, ACTITUDES Y PRÁCTICA EN SALUD.

Correlation between knowledge on dental ergonomics postures, positions of work and postural pain according to response of anatomic zones during clinical practices of dental students.

ABSTRACT

The objective of this study was to determine the correlation between the level of knowledge to different kinds of dental ergonomics postures, work postures and postural pain according to response of anatomic areas during clinical practices of fifth-year students from the School of Dentistry of Peruvian University Cayetano Heredia. The instruments used for this study were: a questionary that evaluated the knowledge on ergonomic working postures, a posture verification list that showed the student's clinical working postures supported by digital photographs analyzed by the Autocad and Corel Draw computer programs. Visual Analogical Scale was used for perception and intensity of postural pain related to anatomic response areas. The Spearman's Rank Correlation Coefficient was used for the statistical analysis. Correct posture was observed in only $22.3 \%$ of the sample. The level of knowledge about dental ergonomic was (37.5\%). The perception of postural pain was higher in the cervical area (75\%), and lower in forearms (15\%). Correlation was found between the level of knowledge about ergonomic dental postures and the application of dental work postures. It is concluded that there is a direct relation between the variables studied.

Keywords: HUMAN ENGINEERING / OCCUPATIONAL HEALTH / DENTAL STUDENTS / HEALTH KNOWLEDGE, ATTITUDES, PRACTICE.
Nadia Verenna Bendezú Aguirre $^{1}$

Edgar Valencia Tapia² Luis Angel Aguilar Mendoza ${ }^{3}$ Cecilia Vélez Fonseca ${ }^{4}$

Cirujano Dentista

${ }^{2}$ Especialista en Psicología de la Ingeniería. Facultad de Psicología.

${ }^{3}$ Coordinador del Área de Neurociencia. Dpto. de Ciencias Morfológicas. Facultad de

Medicina.

${ }^{4}$ Docente del Departamento Académico de

Clínica Estomatológica.

\section{Correspondencia}

Nadia Verenna Bendezú Aguirre Calle D-8. Urb. La Inmaculada - Lima 33, Perú Teléfono: 2853736.

e-mail: bende_nad@yahoo.es

Aceptado para publicación :

17 de julio del 2006

\section{Subvención del estudio}

Premio anual a los mejores proyectos de tesis "Barbara Ann Kotowski de Tejada "- 2004. Universidad Peruana Cayetano Heredia

\section{Introducción}

La expresión enfermedad profesional se refiere a las alteraciones o lesiones corporales que puede sufrir el profesional como consecuencia del desem- peño de su profesión y en el ámbito de su trabajo (1).

Dentro de las enfermedades ocupacionales del odontólogo, las de mayor incidencia son los desórdenes múscu- lo-esqueléticos. Se ha comprobado que inadecuadas posturas de trabajo del odontólogo, pueden dar lugar a afecciones 0 trastornos músculos esqueléticos y vasculares. 
Los dolores de espalda, ciática y hernia discal son signos y síntomas habituales del odontólogo (2-4). El dolor de espalda puede tener un origen psíquico, ya sea en su génesis o su evolución, no debe ser infravalorado, pues suele acompañarse en cuadros de ansiedad, depresión y trastornos psicosomáticos (5).

En relación a los trastornos y/o patologías musculoesqueléticas, es común la degeneración de los discos de la región cervical (6). Ylipaa et al. (7), encontraron que las molestias mioesqueléticas aumentaban con la edad y en la parte baja del brazo derecho y los dos hombros con los años de profesión. Encontraron también bursitis, hipertrofia muscular y contractura muscular fisiológica en odontólogos. Saquy y Djalma (6), mencionan la desigualdad en la altura de los hombros en los odontólogos, así como la tenosinovitis y artritis de las manos. Martin (8), mencionó lesiones por esfuerzos posturales repetitivos, tendinitis y condilitis, síndrome de tensión del cuello, síndromes compresivos del miembro superior. La artrosis patología que más incide en la articulación intervertebral del odontólogo (9). Entre otras patologías frecuentes citaremos la contractura muscular, espondiloartrosis y otras menos frecuentes como espondilolistesis y espondilolísis (10). La repetición de inclinaciones anómalas en relación a los planos sagital y frontal del tronco ocurre con mucha frecuencia en las posturas clínicas del cirujano dentista, estas originarían escoliosis, combinada o no con la cifosis o lordosis lumbar torsiones o giros vertebrales (11).

La Organización Internacional de Estandarización (ISO), define a la ergonomía como una adaptación de las condiciones de trabajo y de vida a las características anatómicas, fisiológicas y psicológicas del hombre en relación a su entorno físico, sociológico y tecnológico (12).

La ergonomía tiene diferentes denominaciones: En Europa: Ergonomía, En los EEUU: Psicología de la ingeniería y Human factors, y en Latinoamérica: Psicología de la Ingeniería. (13).
Psicología de la ingeniería es una disciplina dentro de la psicología, que tiene como objetivo general, el estudio de los procesos de interacción Hombremáquina/sistema, ésta aplica una perspectiva psicológica a los problemas de diseño de equipos, teniendo como objetivo reducir errores, incrementar la productividad y mejorar la calidad de trabajo del operador (13).

El propósito del presente estudio fue estudiar la correlación existente entre el nivel de conocimientos sobre posturas odontológicas ergonómicas, posturas de trabajo y dolor postural según zonas de respuesta, durante las prácticas clínicas de estudiantes de la Facultad de Estomatología Roberto Beltrán Neira.

\section{Materiales y método}

En este estudio descriptivo correlacional participaron 20 estudiantes del quinto año de la Facultad de Estomatología Roberto Beltrán Neira de la Universidad Peruana Cayetano Heredia que realizaron el internado clínico durante el periodo de setiembre-diciembre del 2004, diestros, sin antecedentes de problemas médicos de la columna vertebral.

Se utilizaron unidades dentales, modelo Landus VZ Opti LDF (Gnatus) totalmente eléctricas certificadas conforme norma NBR ISO 6875.

Para el estudio se aplicaron las siguientes técnicas:

Observación postural. La metodología incluyó dos partes: la verificación postural y la validación fotográfica, obteniendo ocho observaciones por alumno.

Verificación postural. Se utilizó una lista de verificación postural compuesta de 10 puntos basada en el BHOP (Balanced Human Operating Position) del Human Perfomance Institute (HPI) de Atami (Japón), compendio de posturas de trabajo odontológico recomendado y aceptado por la Organización Mundial de la Salud (OMS) (10).

Validación fotográfica. Se tomaron fotografías a cada sujeto, durante sus actividades clínicas utilizando una cámara digital (Sony modelo Cyber-shot de 3.2 megapixeles con 2X optical zoom $\mathrm{f}=6.3-12.6 \mathrm{~mm} \mathrm{1:3.8).} \mathrm{Las} \mathrm{fotografías} \mathrm{fue-}$ ron sometidas a análisis de imagen computarizado por medio de los programas Canvas 7.0, Autocad 2000 y Corel Draw 12.

Cuestionario de conocimientos sobre posturas odontológicas ergonómicas.

Se elaboró un cuestionario, de 15 preguntas, basado en el BHOP.

La prueba se construyó con una estructura binaria, por cuanto, a pesar de que contaba con cuatro alternativas de respuesta, sólo una era la correcta.

Éste cuestionario estuvo destinado a obtener información de los sujetos comprendidos en el estudio sobre posturas odontológicas ergonómicas.

Escala Analógica Visual de percepción e intensidad del dolor postural según zonas de respuesta.

Para medir la percepción e intensidad del dolor se utilizó una escala continua, basada en la presencia o ausencia de dolor, que constó con seis zonas de respuesta: zona cervical (cuello), zona dorsal y lumbar (espalda), manos, brazo, antebrazo (miembros superiores), y en caso de aquellos que tenían respuestas positivas para el mismo, se procedió a verificar la intensidad de dolor de cada zona, a través de una escala numérica de la EscalaAnalógica Visual (EAV) del 0 (ausencia de dolor) al 10 (el dolor intenso).

Para el análisis estadístico de estos resultado y determinar si existe una relación estadísticamente significativa, se utilizó el coeficiente de correlación de rangos de Spearman.

\section{Resultados}

El presente estudio, ratifica y pone de manifiesto la situación postural de los alumnos de pre-grado de Estomatología de la Universidad Peruana Cayetano Heredia durante sus actividades clínicas.

De las 1600 observaciones posturales totales que se realizaron, sólo 357 (22,3\%) presentaron posturas correctas (Tabla 1).

De un universo de 240 preguntas del cuestionario de conocimientos sobre posturas odontológicas ergonómicas, sólo 90 (37,5\%) fueron respondidas correctamente (Tabla 2).

En cuanto a los resultados de per- 
cepción del dolor postural según zonas anatómicas de respuesta, la zona cervical obtuvo mayor presencia de dolor con un 75\%, seguido de la zona Lumbar 70\%, Zona dorsal 50\% (Tabla 3).

En cuanto a los resultados de grados de intensidad (EVA) de dolor postural, según zona anatómica tenemos que: La zona cervical, con mayor presencia de dolor postural (75\%) presentó: Intensidad de Grado 4 (20\%). La zona lumbar, con presencia de dolor del $70 \%$ e intensidad de grado 4 (25\%). En la zona dorsal (50\%) e intensidad de grado 4 (30\%).En manos (40\%) e intensidad grado 4 (15\%). En brazos y hombros (40\%), grado 3 (15\%).En antebrazos (15\%), grado 4,5 y 7 (5\%) respectivamente (Tabla 4$)$

La Tabla 5 exhibe resultados en cuanto al análisis del comportamiento del dolor postural según zonas de respuesta. Para efectos de los resultados de información de dolor se consideró a la zona de manos, antebrazos y brazos hombros dentro del rubro de miembros superiores. En cuanto a desde cuando sufre de dolor: En la zona cervical, con resultados de percepción dolorosa más significativos, el $40 \%$ afirmó sufrir de dolor "hace meses". La alternativa "hace meses" también fue la más frecuente en miembros superiores con un 63,64\%, y zona lumbar con un 53,85\%. En cuanto a la forma de inicio: En la zona cervical fue "gradual" en 60\% de los encuestados. La alternativa "súbita" fue respondida mayoritariamente por el 63,64\% en miembros superiores, 53,85\% en la zona lumbar y $50 \%$ en la zona dorsal.

En relación con la presencia del dolor: Para la zona cervical el dolor está presente "sólo en parte del día" en un 73,33\% de los encuestados, el 72,73\% para miembros superiores, $70 \%$ para la zona dorsal y 69,23\% para la zona lumbar. En cuanto a si algunos hechos y/o alternativas le alivian el dolor: Para la zona cervical la alternativa "reposo" fue contestada por el $66,67 \%$ de los encuestados. La opción "reposo" también fue mayoritaria en miembros superiores con el 72,73\% de los encuestados, en la zona dorsal con $80 \%$ y la zona lumbar en el 69,23\%.

En la zona cervical, el $40 \%$ de los encuestados afirmó haber utilizado alguna forma de tratamiento para calmar su dolor. De igual forma, en miembros superiores con $27,27 \%$, zona dorsal con un $50 \%$ y zona lumbar con $30,77 \%$. En cuanto a la interferencia del dolor con sus actividades: el dolor en la zona cervical interfiere con actividades "clínicas y/o laborales" en el 73,33\% de los encuestados. El dolor tanto en miembros superiores, zona dorsal y lumbar también interfieren con las actividades "clínicas y/o laborales" en el $63,64 \%$; 90\% y 69,23\% de los encuestados respectivamente.

Cuando se encuentra preocupado tenso o deprimido el dolor en la zona cervical "aumenta" para el 93,33\% de los encuestados. El dolor en miembros superiores, zona dorsal y lumbar también "aumenta" para el 90,91\%; 50\% y 61,54\% respectivamente. En cuanto a disminuye su dolor la alternativa "cuando pasa ratos agradables" fue contestada por el $100 \%$ en miembros superiores, el 93,93\% en la zona cervical, 100\% en la zona dorsal y $100 \%$ para la zona lumbar. El dolor empeora con cambios de postura para el $100 \%$ de los encuestados sólo en las zonas dorsal y lumbar.

La Tabla 6 exhibe las correlaciones generales entre el resultado global de Nivel de conocimientos con posturas de trabajo por medio del coeficiente de correlación de rangos de Spearman obtuvieron un valor de correlación estadísticamente significativa de $+0,494$ (p al 0,05>0,44). Las correlaciones generales entre resultado global de posturas de trabajo y percepción del dolor postural, no obtuvieron un valor de correlación estadísticamente significativo de $+0,01$ ( $p$ al 0,05>0,44). Las correlaciones generales entre el resultado global de nivel de conocimientos y percepción del dolor postural, no obtuvieron un valor de correlación estadísticamente significativo -0,213 (p al 0,05>0,44).

Tabla 1. Resultados generales de posturas de trabajo odontológico - lista de verificación postural

\begin{tabular}{|c|c|c|c|}
\hline \multicolumn{2}{|c|}{ Verificación postural } & \multirow{2}{*}{$\mathrm{n}$} & \multirow{2}{*}{$\frac{\%}{35,0}$} \\
\hline I. & Situado el paciente en decúbito supino el eje de su columna vertebral será paralelo al eje horizontal. & & \\
\hline II. & El operador se encuentra sentado; su columna vertebral debe estar perpendicular en relación a la & & \\
\hline & columna del paciente. & 19 & 11,9 \\
\hline III. & $\begin{array}{l}\text { Las piernas del operador estarán un poco separadas, de forma que uniendo con líneas imaginarias } \\
\text { el cóccix y las rótulas formen un triángulo equilátero, en cuyo centro geométrico se encontrará }\end{array}$ & & \\
\hline & la boca del paciente (triángulo fisiológico de sustentación) & 11 & 6,9 \\
\hline \multirow{2}{*}{ IV. } & La flexión de las rodillas y la altura del taburete serán tales que las piernas y ante-piernas del & & \\
\hline & operador formen un ángulo recto. & 24 & 15,0 \\
\hline $\mathrm{V}$. & Pierna y pies del operador en ángulo recto. & 33 & 20,6 \\
\hline \multirow[t]{2}{*}{ VI. } & Total apoyo plantar con disposición paralela entre ellos, sin mostrar inclinaciones que determinen & & \\
\hline & apoyo sobre las líneas internas o externas de los pies. & 3 & 1,9 \\
\hline VII. & Manos y dedos serán los puntos de apoyo sobre el campo de trabajo & 99 & 61,9 \\
\hline VIII. & Flexión cervical mínima con cabeza ligeramente inclinada. & 47 & 29,4 \\
\hline IX. & Brazos lo menos alejados del eje vertical (columna del operador). & 54 & 33,8 \\
\hline $\mathrm{X}$ & Línea imaginaria que cruza ambos hombros del operador deberá ser lo más paralela al piso. & 11 & 6,9 \\
\hline \multicolumn{2}{|c|}{ Total de observaciones correctas } & 357 & 22,3 \\
\hline \multirow{2}{*}{\multicolumn{2}{|c|}{$\begin{array}{l}\text { Total de observaciones incorrectas } \\
\text { Universo de observaciones posturales }\end{array}$}} & 1243 & 77,7 \\
\hline & & 1600 & 100,0 \\
\hline
\end{tabular}

$\mathrm{n}$ : observaciones correctas 
Tabla 2. Resultados generales de nivel de conocimientos sobre posturas odontológicas ergonómicas

Preguntas del cuestionario de conocimientos

n $\%$

1. Secuencia correcta de la columna vertebral: cervical, dorsal, lumbar, sacra.

2. Eje horizontal o línea del suelo, y el eje vertical o columna del operador: perpendiculares $\left(90^{\circ}\right)$.

$10 \quad 50,0$

3. Paciente en decúbito supino, eje de columna vertebral: $0^{\circ}$ con respecto a la horizontal.

4. Operador sentado, columna vertebral: Paralela con respecto a la vertical y perpendicular a la columna del paciente.

5. El operador sentado: mantendrá las piernas separadas.

En esta posición se trazan líneas imaginarias que unirán el

cóccix y las dos rótulas .Las líneas trazadas formarán un triángulo equilátero, donde la boca del paciente se encontrará en el centro geométrico del triángulo.

6. Operador sentado, hay flexión de las rodillas debido a la altura del taburete: piernas y ante-piernas formarán un ángulo de $90^{\circ}$.

7. Operador sentado: piernas y pies: ángulo de $90^{\circ}$.

8. Operador sentado: codos flexionados de tal forma que brazos y antebrazos: ángulo de $90^{\circ}$.

9. Flexión cervical del operador sentado: mínima con cabeza ligeramente inclinada.

Los brazos en relación con parrilla costal del operador (sentado): ángulo de $0^{\circ}$.

11. La cabeza del paciente en relación con el operador (sentado):

En línea media sagital del operador.

2. Línea imaginaria que cruza ambos hombros del operador: paralela al piso.

Total de respuestas correctas

Total de respuestas incorrectas

Universo de respuestas

$\mathrm{n}$ : observaciones correctas

Tabla 3. Resultados generales de percepción del dolor postural según zonas de respuesta.

\begin{tabular}{lrc}
\hline Zonas de respuesta & $\mathrm{n}$ & $\%$ \\
Manos & 8 & 40,0 \\
Antebrazos & 3 & 15,0 \\
Brazos y hombros & 8 & 40,0 \\
Zona cervical & 15 & 75,0 \\
Zona dorsal & 10 & 50,0 \\
Zona lumbar & 14 & 70,0 \\
\hline Presencia del dolor postural total & 58 & 48,3 \\
Ausencia del dolor postural total & 62 & 51,7 \\
Universo de percepción de dolor postural & 120 & 100,0 \\
\hline
\end{tabular}

$\mathrm{n}$ : presencia de dolor postural

Tabla 4. Resultados de percepción e intensidad del dolor según zonas de respuesta.

\begin{tabular}{|c|c|c|c|c|c|c|c|c|c|c|c|c|}
\hline \multirow[b]{2}{*}{$\begin{array}{l}\text { Zonas de } \\
\text { respuesta }\end{array}$} & \multicolumn{11}{|c|}{ Grados de intensidad (EAV) } & \multirow{2}{*}{$\begin{array}{l}\text { presencia } \\
\text { de dolor } \\
\%\end{array}$} \\
\hline & $\begin{array}{l}0 \\
\mathrm{n}(\%)\end{array}$ & $\begin{array}{l}1 \\
\mathrm{n}(\%)\end{array}$ & $\begin{array}{l}2 \\
\mathrm{n}(\%)\end{array}$ & $\begin{array}{l}3 \\
\mathrm{n}(\%)\end{array}$ & $\begin{array}{l}4 \\
\mathrm{n}(\%)\end{array}$ & $\begin{array}{l}5 \\
\mathrm{n}(\%)\end{array}$ & $\begin{array}{l}6 \\
\mathrm{n}(\%)\end{array}$ & $\begin{array}{l}7 \\
\mathrm{n}(\%)\end{array}$ & $\begin{array}{l}8 \\
\mathrm{n}(\%)\end{array}$ & $\begin{array}{l}9 \\
\mathrm{n}(\%)\end{array}$ & $\begin{array}{l}10 \\
\mathrm{n}(\%)\end{array}$ & \\
\hline Manos & $12(60)$ & $1(5)$ & 0 & $2(10)$ & $3(15)$ & 0 & $1(05)$ & 0 & $1(05)$ & 0 & 0 & 40 \\
\hline Antebrazos & $17(85)$ & 0 & 0 & 0 & $1(05)$ & $1(05)$ & 0 & $1(5)$ & 0 & 0 & 0 & 15 \\
\hline Brazos y hombros & $12(60)$ & 0 & $2(10)$ & $3(15)$ & $1(05)$ & $2(10)$ & 0 & 0 & 0 & 0 & 0 & 40 \\
\hline Zona cervical & $05(25)$ & 0 & $2(10)$ & 0 & $4(20)$ & $3(15)$ & $2(10)$ & $1(5)$ & $2(10)$ & $1(5)$ & 0 & 75 \\
\hline $\begin{array}{l}\text { Zona dorsal } \\
\text { (Tórax posterior) }\end{array}$ & $10(50)$ & 0 & 0 & 0 & $6(30)$ & $2(10)$ & $1(05)$ & 0 & $1(05)$ & 0 & 0 & 50 \\
\hline $\begin{array}{l}\text { Zona lumbar } \\
\text { (Cintura posterior) }\end{array}$ & 06(30) & $1(5)$ & 0 & $2(10)$ & $5(25)$ & $3(15)$ & $2(10)$ & 0 & $1(05)$ & 0 & 0 & 70 \\
\hline
\end{tabular}

EAV:escala analógica visual 
Tabla 5. Resultados en relación al comportamiento del dolor postural según zonas de respuesta.

\begin{tabular}{|c|c|c|c|c|c|}
\hline & & MS & $\mathrm{ZC}$ & ZD & $\mathrm{ZL}$ \\
\hline Desde cuando & horas & - & 20,00 & 10,00 & 0,00 \\
\hline \multirow[t]{3}{*}{ sufre de dolor } & días & 36,36 & 26,00 & 50,00 & 38,46 \\
\hline & meses & 63,64 & 40,00 & 30,00 & 53,85 \\
\hline & años & - & 13,33 & 10,00 & 7,69 \\
\hline \multirow[t]{2}{*}{ Forma de inicio } & súbita & 63,64 & 40,00 & 50,00 & 53,86 \\
\hline & gradual & 36,36 & 60,00 & 50,00 & 46,15 \\
\hline El dolor está & a lo largo de todo el día & 9,09 & 6,67 & - & 0,00 \\
\hline \multirow[t]{3}{*}{ presente } & solo en parte del dia & 72,73 & 73,33 & 70,00 & 69,23 \\
\hline & en parte de la semana & 9,09 & 6,67 & 10,00 & 15,38 \\
\hline & ocasionalmente cada mes & 9,09 & 13,33 & 20,00 & 15,38 \\
\hline Alguno de los & reposo & 72,73 & 66,67 & 80,00 & 69,23 \\
\hline hechos siguientes & sueño & 9,09 & 26,67 & 10,00 & 7,69 \\
\hline le alivia el dolor & cambios de postura & 18,18 & 6,67 & 10,00 & 23,08 \\
\hline Utilizó alguna & si & 27,27 & 40,00 & 50,00 & 30,77 \\
\hline forma de tratamiento & no & 72,73 & 60,00 & 50,00 & 69,23 \\
\hline Interfiere su dolor & domésticas & 18,18 & 6,67 & 10,00 & 15,38 \\
\hline \multirow[t]{2}{*}{ con sus actividades } & laborales y/o clínicas & 63,64 & 73,33 & 90,00 & 69,23 \\
\hline & social y/o recreativas & 18,18 & 20,00 & 0,00 & 15,38 \\
\hline Cuando usted está & aumenta & 90,91 & 93,33 & 50,00 & 61,54 \\
\hline preocupado, tenso ó & disminuye & 0,00 & 0,00 & 0,00 & - \\
\hline deprimido, su dolor & no varía & 9,09 & 6,67 & 50,00 & 38,46 \\
\hline Disminuye su dolor & está atareado & - & 6,67 & 0,00 & 0,00 \\
\hline cuando usted & pasa ratos agradables & 100,00 & 93,33 & 100,00 & 100,00 \\
\hline \multirow[t]{3}{*}{ Empeora su dolor con } & cambios de postura & - & - & 100,00 & 100,00 \\
\hline & tos estornudos, esfuerzos & - & - & 0,00 & 0,00 \\
\hline & comer y/o beber & - & - & 0,00 & 0,00 \\
\hline
\end{tabular}

MS:miembros superiores. ZC:zona cervical. ZD:zona dorsal. ZL:zona lumbar

Tabla 6. Matriz de correlaciones de resultados globales (coeficiente de correlación de rangos de Spearman).

\begin{tabular}{lllll}
\hline correlaciones generales & & " r " & p 0,05 & ES \\
nivel de conocimientos & postura de trabajo & 0,494 & 0,44 & si \\
postura de trabajo & dolor postural & 0,010 & 0,44 & no \\
nivel de conocimientos & dolor postural & $-0,213$ & 0,44 & no \\
\hline
\end{tabular}

nivel de significancia (n: 20 ; gl: 18). $\mathrm{p}<0,05$

\section{Discusión}

El presente estudio ratifica y pone de manifiesto la situación postural de los alumnos de pre-grado de Estomatología de la Universidad Peruana Cayetano Heredia durante sus actividades clínicas.

Sólo el 22.3\% de los sujetos presentaron posturas correctas, a pesar de ser alumnos del último año de estudio. Estudios previos sobre postura en Odontólogos fueron realizados también por Al Wazzan et al. (11) quienes refieren la existencia de problemas posturales en cuello y espalda. Nuestros resultados evaluaron también estas zonas pero con enfoques distintos, gracias al Balanced Human Operating Position (BHOP), recomendada por expertos en salud oral de la Organización Mundial de la Salud - OMS (10). La investigación incluyó ítems de la misma, facilitado por medio de una lista de verificación postural, con zonas anatómicas referenciales tales como: zona cervical, miembros superiores y espalda, intrínsecos a los ítems de postura.

El apoyo plantar con disposición paralela de los pies fue efectivo en sólo un $1.9 \%$ de las observaciones, esto supone un alto índice de desequilibrio postural, considerando el principios de máximo equilibrio (BHOP), una posición estable requiere de amplia base de sustentación a nivel del suelo. Supone también riesgos de alteraciones del sistema circulatorio (varices, edemas, dolor e inflamación) por compresión mus- cular, en extremidades inferiores, evitando el retorno venoso (9). Mientras que el ítem $\mathrm{N}^{\circ}$ VII "manos y dedos serán los puntos de apoyo sobre el campo de trabajo" refirió el $61.9 \%$ de posturas correctas, siendo el puntaje más alto de la lista de verificación postural.

Se registró también información adicional en cuanto a procedimientos clínicos por especialidad, durante todas las observaciones: rehabilitación oral (40\%), endodoncia (25\%), operatoria dental (18\%). Lotte et al. (14), no encontraron diferencias significativas entre procedimientos odontológicos más comunes (examen dental, profilaxis y operatoria dental) con relación a tipos de posturas, frecuencias de movimiento $o$ actividad muscular. 
Los procedimientos de Rehabilitación oral y endodoncia, posiblemente requieran de mayor precisión y visibilidad (preparación dentaria e instrumentación), pudiendo degenerar en posturas forzadas e inadecuadas.

De igual forma, en el $71.88 \%$ de las observaciones efectuadas, se constató ausencia de asistentas dentales. La presencia de asistentes dentales puede influenciar en el desempeño del trabajo odontológico, debido a la reducción de movimientos (al sujetar y alcanzar el instrumental), por medio de la técnica a cuatro manos. Así mismo, permite un trabajo efectivo, en mejores condiciones y por un tiempo más prolongado con el paciente (10).

Los conocimientos sobre posturas ergonómicas durante el trabajo odontológico son fundamentales a nivel de pre-grado, por estar en proceso de formación, coadyuvando con la prevención primaria de lesiones posturales o músculo-esqueléticas incluidas dentro de las enfermedades ocupacionales del odontólogo, considerando que el $62.5 \%$ de las preguntas sobre conocimientos de posturas odontológicas ergonómicas fueron respondidas incorrectamente.

En cuanto a los resultados de percepción del dolor postural nuestros resultados fueron casi similares a los encontrados por López (5), que refiere dolor más frecuente en zona del cuello, (79.4\%). por la sobrecarga anatómica y fisiológica que sufre la columna vertebral al trabajar sentados con la espalda inclinada hacia delante y por la mayor movilidad de estas zonas. Para Kendall (15), el dolor es también influenciado por tensión de los brazos al trabajar en extensión, separados del tronco y elevados. Sin embargo, la posición encontrada en las observaciones en relación a uno de los ítems "Brazos lo menos alejados del eje vertical-columna del operador" fue efectiva en sólo un 33.8\%. No descartar otras causas de dolor postural tales como: patología inflamatoria, infecciosa, neoplásica, factores psicológicos que no fueron objeto del presente estudio, recomendable para estudios posteriores.
Al Wazzan et al. (11), encontraron un $54.4 \%$ de dolor de cuello o cervical. Osborn (16), encontró que las zonas más frecuentes de dolor en los odontólogos son las vértebras cervicales. Saquy Djalma (6), refieren que es común la degeneración de los discos intervertebrales cervicales. Kendall's (15) menciona que trabajar con la cabeza inclinada y espalda arqueada produce tirantez de los músculos posteriores del cuello (trapecio), compresión posterior de las carillas articulares y debilidad en los cervicales anteriores. Los resultados ponen de manifiesto la presencia de dolor desde etapas tempranas de formación, ya que la población estuvo conformada por sujetos jóvenes. Los resultados encontrados en sujetos jóvenes, fueron casi similares al de poblaciones adultas o con mayor antigüedad en el ejercicio de la profesión, estudiadas por los autores anteriormente mencionados. Pudiéndose desprender, que la presencia de dolor cervical es una constante tanto en poblaciones jóvenes como adultas.

El 70\% presentó dolor en zona lumbar, resultado superior al 59.8\% encontrado por López (5). En el 85\% de las observaciones posturales realizadas, presentaron ausencia de respaldo lumbar. Según Carrillo (10), al no haber respaldo lumbar, la presión a nivel de los discos intervertebrales aumenta y la musculatura vertebral realiza mayor esfuerzo para contrarrestar la cifosis de la columna. Según Osborn et al. (16), las zonas más frecuentes de dolor postural en odontólogos son las vértebras lumbares.

Los resultados del dolor en la zona dorsal (50\%) fueron coincidentes con los encontrados por López (5) con $50.5 \%$ y Marshal et al. (17) con 59\%.

El dolor de manos estuvo presente en un $40 \%$. Al respecto Saquy et al. (6), mencionan la tenosinovitis y artritis de las manos; y Kendal (15) la aparición del síndrome del túnel carpiano.

Para el dolor en brazos y hombros, López (5) refiere un 58.3\% de dolor de hombros. Precisamos que nuestros resultados (40\%) consideran en un solo rubro a brazos y hombros, mientras que López lo consideró de manera independiente.
Ylipaa et al. (7), las molestias mioesqueléticas en la parte baja del brazo derecho y hombros aumentan con la edad y años de profesión. Saquy et al. (6), menciona bursitis, hipertrofia muscular, contractura muscular fisiológica en miembros superiores, desigualdad en altura de hombros, tenosinovitis y artritis de las manos, comunes en odontólogos. Nuestros resultados señalan la existencia de inclinación de hombros tanto, hacia el lado derecho, como izquierdo, en el 73,75\% de las observaciones totales efectuadas.

Los resultados de grados de intensidad (EVA) de dolor postural según zonas anatómicas fueron significativos. Es importante realzar el aporte de la presente investigación, dado que no se encontraron estudios y referencias bibliográficas respecto al estudio de la Intensidad del dolor postural en odontólogos según zona anatómicas.

En cuanto al análisis del comportamiento del dolor postural según zonas de respuesta. La referencia mayoritaria de los sujetos en cuanto al alivio del dolor cervical durante el "reposo" confirma que éste dolor es de tipo mecánico, tal como lo afirma López (5), con aparición de predomino diurno, en relación con la función de la estructura afectada, mejora con el reposo y aumenta con la actividad.

Así mismo, el 40\% de los encuestados afirma haberse sometido a fisioterapia y/o tratamiento farmacológico por medio de anti-inflamatorios para aliviar su dolor, una cifra nada despreciable, que no sólo indicaría que el problema es de cierto grado de consideración, sino que estaría influyendo en la salud y estilo de vida de los operadores. En consecuencia, un innecesario desembolso económico, de haberse podido tomar medidas preventivas en cuanto a hábitos de postura de trabajo ergonómicos.

El dolor en todas las zonas anatómicas aumentó considerablemente cuando los individuos se encontraban "preocupados o deprimidos", y disminuyó en igual proporción cuando "pasaban ratos agradables". Confirmando lo expuesto por López (5) respecto a los fac- 
tores psicológicos asociados con el dolor postural, muchos de evolución y/ u origen emotivo.

El dolor postural empeora con cambios de postura para el 100\% de los encuestados sólo en las zonas dorsal y lumbar. Según Mc Culloh (18) está ligado con esfuerzos, tensiones excesivas, desórdenes o patologías músculoesqueléticas como consecuencia de posturas incorrectas. Navarro et al. (19), afirman que el dolor postural puede asociarse con un desequilibrio en las arcadas dentarias ya sea por falta de piezas o mal posiciones dentarias. Se recomienda investigar la relación entre el estado oclusal y la presencia del dolor postural.

El coeficiente de correlación positivo $(+0,494)$ entre el resultado global de la prueba de nivel de conocimiento de posturas odontológicas ergonómicas, y el resultado global de observación considerados en la lista de verificación postural, revela una relación directa entre ambas variables estudiadas: "a mayor nivel de conocimientos de posturas odontológicas ergonómicas, mayor presencia de posturas correctas de trabajo, y a un menor nivel de conocimientos de posturas odontológicas ergonómicas, menor presencia de posturas correctas de trabajo".

A pesar de que los resultados de las demás correlaciones no obtuvieron valores estadísticamente significativos se observa un bajo nivel de conocimiento sobre aspectos ergonómicos en odontología y falta de control postural. De igual forma, la presencia de dolor postural y sus implicancias durante las actividades clínicas del alumno de Estomatología. Los resultados nos indican que se necesitará reforzar a los alumnos respecto al conocimiento, así como, la concientización y utilización de medidas preventivas en el desarrollo de su práctica clínica.

\section{Conclusiones}

- El nivel de conocimientos sobre posturas odontológicas ergonómicas de trabajo registrado en los alumnos, fue bajo.

- La ejecución de posturas de trabajo registradas durante las prácticas clínicas odontológicas fue mayoritariamente incorrecta.

- La presencia de dolor postural fue mayor en la zona cervical (75\%).

- Con respecto al nivel de conocimientos sobre posturas odontológicas ergonómicas y la ejecución de posturas de trabajo, se encontró correlación estadísticamente significativa.

- Con respecto a la ejecución de posturas de trabajo y la percepción e intensidad del dolor postural según zonas de respuesta, no se encontró correlación estadísticamente significativa.

- Con respecto al nivel de conocimientos sobre posturas odontológicas ergonómicas y la percepción e intensidad del dolor postural según zonas de respuesta, no se encontró correlación estadísticamente significativa.

\section{Referencias bibliográficas}

1. Osorio Ruiz ME. Enfermedades profesionales en odontoestomatología. Rev Prof Dent 2001; 4(1):39.

2. Carrillo J, Calatayud J, Álvarez C, Carrillo P. Algunos aspectos sobre ergonomía y su aplicación en Odontología. España: Ilustre Colegio Oficial de Odontólogos y Estomatólogos de la I Región; 1992. p.12-13.

3. Carrillo P, Casado I. Posiciones y posturas de trabajo del odontólogo y del auxiliar. Rev Gaceta Dental 2001; 114:48-57.

4. Monastrio Vicente JL, et al. El dolor de espalda en la práctica Odontoestomatológica. Rev Euro Odont 1989; 1:89-96.

5. López M. Desórdenes músculo esqueléticos y su relación con el ejercicio profesional en odontología. Rev Gaceta Dental 2003; 139:15-18.

6. Saquy PC, Djalma JP. Cómo prevenir las enfermedades ocupacionales. Rev Mundo Odont 1994; 2(7):20.
7. Ylipaa V. Physical and psychosocial work enviroment among swedish dental hygienist: risk indicators for musculoskeletal complaints. Swed Dent J 1997; 21(3):111.

8. Martin Filho F. Lessôes por esforços repetitivos. J Odont Focus Brasil 1999; 1(27).

9. Toledano M, Osorio R. El Manual de odontología-enfermedads profesionales del odontoestomatólogo. Barcelona: Masson SA; 1995.

10. Carrillo P. Estudio de prevención de las lesiones posturales de la espalda en el odontólogo. Rev Gaceta Dental 2003; 137:60-74

11. Al Wazzan KA, Almas K, Al Shethri Se, et al. Back \& Neck problems among dentists and dental auxiliaries. J Contemp Dent Pract 2001; 3(2):17-30.

12. Carrillo JS. Ergonomía en odontología: Planteamiento de necesidades. Rev Profesión Dental 2001; 4(4):27-8.

13. Wickens CD, Gordon SE, Liu Y. An introduction to human factor engineering. New York: Longman; 1998.

14. Lotte F, Christensen H, Bakke M. Musculoeskeletal disorders among dentists and variation in dental work. J Applied Ergon Great Brit 1998; (2)29:119-25.

15. Kendall's FP. Músculos, pruebas, funciones y dolor postural. Madrid: Marban libros; 2000.

16. Osborn JB, Newell KJ, Rudney JD. Musculoeskeletal symptoms in New South Wales dentists. Aust Dent J 1997; 42:240-6.

17. Marshall ED, Duncombe LM, Robinson RQ, Kilbreath SL. Musculoskeletal symptoms in New South Wales dentists. Aust Dent J 1997; 42(4):240-6.

18. Mc Culloch J. Macnab’s backache. 3 ed. Maryland: Williams \& Wilkins CO; 1997.

19. Navarro C, Ratia F, Sánchez F. El dolor de espalda causado por malposiciones dentarias. Rev Gaceta Dental 2003; 135:17-19. 